\title{
Strategy Communication and Measurement Systems
}

\author{
Pascal Courty ${ }^{1,2}$ \\ Department of Economics \\ Universitat Pompeu Fabra
}

October 1, 1997

\begin{abstract}
Organizations often face the challenge of communicating their strategies to local decision makers. The difficulty presents itself in finding a way to measure performance which meaningfully conveys how to implement the organization's strategy at local levels. I show that organizations solve this communication problem by combining performance measures in such a way that performance gains come closest to mimicking value-added as defined by the organization's strategy. I further show how organizations rebalance performance measures in response to changes in their strategies. Applications to the design of performance metrics, gaming, and divisional performance evaluation are considered. The paper also suggests several empirical ways to evaluate the practical importance of the communication role of measurement systems.
\end{abstract}

JEL: L21, D21.

Keywords: Measurement Systems, Firm Objective, Performance Measurement, Communication.

\footnotetext{
${ }^{1}$ Department of Economics, Universitat Pompeu Fabra, Ramon Trias Fargas 25-27, 08005 Barcelona, Spain. Email courty@upf.es. Tel. (343) 542 2729. Fax. (343) 5421746.

${ }^{2}$ I would like to thank James Heckman, Gerald Marschke, Canice Prendergast, and Sherwin Rosen for helpful comments. I am grateful to participants at the Coleman workshop, and the Simon GSB and Universitat Pompeu Fabra seminars. I acknowledge support from the Sloan Foundation and The University of Chicago. All errors are exclusively mine.
} 


\section{Strategy Communication and Measurement Systems}

October 1, 1997

ABSTRACT: Organizations often face the challenge of communicating their strategies to local decision makers. The difficulty resides in finding the way to measure performance that best translates how the organization's strategy should be implemented at the local level. I show that organizations solve this communication problem by combining performance measures in such a way that performance gains come closest to mimicking value-added as defined by the organization's strategy. I further show how organizations rebalance performance measures in response to changes in their strategies. Applications to the design of performance metrics, gaming, and divisional performance evaluation are considered. The paper also suggests several empirical ways to evaluate the practical importance of the communication role of measurement systems. 
"Those companies that can translate their strategy into their measurement systems are far better able to execute their strategy because they can communicate their objectives and their targets."

Robert Kaplan and David Norton ${ }^{1}$

\section{Introduction}

Many organizations spend large amounts of resources on performance measurement systems to communicate to their employees how to add value. On the other side of this market, consultants have been offering in recent year a wide range of performance metrics that are supposed to link decision making with firm strategy. Kaplan and Norton emphasize in the opening quote that the new focus on the design of these measurement systems is how well they drive behavior by clearly communicating organizations' strategies. ${ }^{2}$ Surprisingly, this communication role of performance measurement system has not received much attention in the organization literature. Although this literature explains why organizations refrain from using incentives to identify and reward good performance, it fails to explain not only the prevalence of performance measurement systems but also their economic functions. ${ }^{3}$ The purpose of this paper is to develop a theory explaining how performance measures are chosen and balanced to communicate organizations' strategies.

To model these issues formally, a stereotypical organization composed of a center and an agent is considered. ${ }^{4}$ The center has superior knowledge about the general strategy of the organization while the agent has superior local knowledge. In addition, the agent's and the organization's knowledge corresponds to what Jensen and Mercklind describe as specific knowledge meaning that it cannot be efficiently communicated. Although the center cannot communicate its strategy explicitly, it can influence local decision making by asking the agent to maximize a set of performance measures. Performance measures

\footnotetext{
${ }^{1}$ Kaplan and Norton (1996), ibid p. 146

${ }^{2}$ See Eccles, 1991, Burns, 1992 and Locke and Latham, 1990.

${ }^{3}$ An exception is Rajagopalan (1996). See Gibbons (1996) for a theoretical and empirical review of the incentive literature.

${ }^{4}$ This theory applies to public and private sector, divisional, production unit or individual performance measures.
} 
are interpreted in this paper as an efficient way of improving decision making by communicating a clear objective. This structure is consistent with the observation that most measurement system do not represent exactly the organization's strategy; rather these systems are an imperfect approximations.

The center combines the performance measures by choosing to put more emphasis, or weight, on some than on others. I restrict attention to linear combinations of performance measures because these schemes are simple and commonly-used. To interpret the optimal linear weights, it is useful to regard the performance measures and the strategy of the organization as functions of the decisions the agent must make. These decisions could be, for example, investing resources in competing projects where each project has its own characteristics which are privately observed by the agent. The optimal linear weights have a regression interpretation: the center combines the performance measures so that performance gains come closest to mimicking value-added as defined by the organization's strategy. Thus, the center puts more weight on those performance measures which better predict the decisions which are most aligned with the organization's strategy. In response, the agent selects and invests more resources in the projects with higher return on the linear combination of performance measures. These projects are also the most valuable to the organization. Performance measures perform well when (a) the agent has more local information and (b) performance gains well predict value-added. To a first order approximation, better proxies for the organization's strategy reduce the cost of using imperfect performance measures proportional to the variations in the value-added explained by the variations in performance gains.

This work contributes to three literatures. First, the management literature emphasizes the importance of selecting performance measures which are consistent with the strategy of an organization. For example, Stewart's popular concept of economic valueadded (1991) combines measures of earning with measures of cost of capital employed. Kaplan and Norton's concept of balanced scorecard (1996) goes one step further and combines external measures for shareholders and customers value with internal measures 
of critical business processes, innovation and learning and growth. Missing from this literature, however, is a formal theory of how to balance different measures. By formally modeling the information asymmetry within an organization, this paper offers a rigorous answer to the problem of selecting and balancing measures to convey a strategy to local decision makers.

Second, this work provides an alternative interpretation to the role of performance measures to the traditional one found in the organization literature (Gibons, 1996). This literature has focused on the incentive aspect of performance measures. Under the received view, the principal chooses financial awards to trade-off hard effort and insurance risk. By focusing on this trade-off, however, this literature ignores the economic role of performance measure of improving local decision-making making by communicating clear objectives. By distinguishing performance measurement systems (the choice of the performance measures) from performance incentive systems (the choice of the awards), this paper identifies two polar economic functions for these systems: communicating a strategy and inducing effort. Toward the end of the paper, I show how these two functions can be reconciled under a general performance weights formula.

Finally, this work contributes to the decentralization literature (see e.g. Weitzman, 1971). I interpret the use of performance measures as a solution to a fundamental problem of communication. This problem was stated in Hayek's seminal article on the use of knowledge in society (Hayek, 1945). Optimal decisions require that decision-makers gather different pieces of information from many agents, implying that communication is necessary to improve decisions. The price system, emphasized by Hayek, facilitates the exchange of information within the marketplace. Organizations must construct some mechanism to reproduce within the organization the role that the price system plays among market players. In this regard, this paper extends Weitzman's study of the comparative advantage of centralized and decentralized organizations by looking at richer communication devices.

The paper proceeds as follows. The next section describes the model and derives the 
optimal balance of performance measures. Section 4 evaluates how performance measures perform. Section 5 extends the model to financial incentives. Application to the design of performance metrics, gaming and divisional performance measurement are discussed toward the end of these sections.

\section{The Optimal Balance of Performance Measures}

Performance measures communicate objectives to local decision makers which often do not perfectly represent the true strategy, or goal, of the organization (Baker, 1992). For example, performance measures often induce unintended behaviors such as gaming (Lawler, 1990 and Courty and Marschke, 1997). This, however, only partially illustrates what I mean by imperfect performance measures. More generally, performance measures rarely perfectly represent contributions to the firm's value. ${ }^{5}$ Because of this lack of perfect and universal proxy, organizations must constantly adjust their measurement systems so that it is aligned with their strategies.

Organizations typically measure performance on several dimensions and balance each of these dimensions. For example, depending on the objective that is communicated, measures of short-term financial performance such as return on capital employed or project profitability are sometimes combined with measures of less tangible assets such as market share, customer satisfaction index, or even employee commitment. This balance between different performance measures is explicit when agents are offered formal performance metrics or scorecards and implicit when agents are informally communicated the emphasis put on each measure.

Often performance measurement systems are coupled with incentive systems which reward performance either financially or through other decisions (e.g. promotion, training). Surprisingly, the fraction of the agent's compensation paid as a financial award are usually low, although it varies widely across compensation systems (Baker, Jensen and

\footnotetext{
${ }^{5}$ Measures of stock market appreciation may constitute valid performance drivers for top management. However, few organizations have traded stocks and top management constitute only a very small fraction of the workforce.
} 
Murphy, 1988). This observation suggests two distinct concepts of weights in performance measure: (a) the relative weights determine the emphasis put on each performance measure; (b) the absolute weights determine how high performers are rewarded and/or low performers punished. These two concepts correspond to the distinction between performance measurement systems and performance incentive systems, which plays a key role in this paper. Most of this paper will study how performance measures are chosen and how they are balanced (the relative weights on performance measures). Toward the end of the paper, I will take into account the incentive dimension of some performance measurement systems (the absolute weights on performance measures).

\subsection{The Model}

Consider a stereotypical organizational structure composed of a center and an agent. The center-agent pair should be taken very generally. For example, it could be the entire organization and its divisions or a division and its business units, and within each unit there could be differentiation between top, mid-level managers, supervisors and employees. The agent invests in a continuum of projects identified by their types $\theta \in \Theta$ representing what Hayek (1945) called the 'knowledge of particular circumstances of time and place'. ${ }^{6}$ Let $q(\theta)$ be the resources invested in project $\theta$. Each project is associated with a value function $v(\theta, q)$ representing the value to the organization as evaluated according to its true strategy. The concept of value should be taken broadly: it represents what is sometimes defined as the value to the stakeholder of the organization. Defined in a narrow sense, the stakeholders are the shareholder but it could also include the organization's customers and employees. The functions $v$ are assumed to be increasing and concave in the investment level. In addition, each project has decreasing returns after some level of investment so that the optimal investment is finite.

$\theta$ is a measure of the informational gap between the center and the agent in the sense that the agent is able to identify the projects' type $\theta$ but does not know the projects'

\footnotetext{
${ }^{6}$ This corresponds to what is known as 'hidden information situations' in the contract literature, where the agent receives private information after signing a contract but before choosing an action.
} 
value functions and the center knows the projects' value functions but does not observe the project types. In addition, the information on the project types and on the value function is too costly to communicate; it corresponds to what Jensen and Merkling (1992) describe as specific knowledge. The value functions, however, can be decomposed into a component which can be communicated, $v^{0}$, and another component which cannot, $v^{1}$, with $v=v^{0}+v^{1} \cdot{ }^{7}$ For example, when accounting cost figures measure well some dimensions of true economic cost, $v^{0}$ would be a measure of these costs.

The center communicates its strategy to the agent through a set of $J$ performance measures, $m^{j}(\theta, q)$ for $j=1 \ldots J$. Let $m$ be the vector of performance measures. The concept of performance measures encompasses a wide range of variables. It includes financial measures of return-on-investment or cash flow, measures of market impact such as gross margin on sales or customer ranking surveys, and measures of internal business practice such as on-time delivery, internal and external employee training, and new product development. For simplicity, the functions $m^{j}$ will be assumed to be increasing and concave in the investment level, with decreasing returns for investments large enough. In the rest of the paper, a performance measure will be defined as the average performance over all projects, $\mathrm{Em}^{j}(\theta, q(\theta))$. The center contracts only over the sum of all performance outcomes. The organization's center knows the joint-distribution, over all projects, of the value function and the performance measures. The focus will be on linear combinations of performance measures because these schemes are simple, and commonly employed. In addition, the concept of weights on performance measures is intuitive because it corresponds to the concept of shadow prices. Finally, I rule out negative performance weights to guaranty that the agent's problem is single peaked. ${ }^{8}$

This way of capturing the informational gap within organizations is fairly standard in the organization literature. As early as 1971, Weitzman used a similar model to study the

\footnotetext{
${ }^{7}$ Although this assumption is not very restrictive, it is necessary to prove the main results of the paper.

${ }^{8}$ This assumption is not very restrictive. For example, it holds under the technical assumption that for each performance measure $j=1 \ldots J$, there exists a non-zero measure set of projects such that for these projects (a) $m^{j}(\theta, \infty)=v(\theta, \infty)=-\infty$, and (b) $m^{k}(\theta, q)$ is finite $\forall k \neq j$.
} 
benefits of decentralization, and more recently, Baker (1992), built up on that structure to study the implications of moral hazard with a risk-neutral agent. It should also be noted that Holmstorm and Milgrom (1991) briefly mention a discrete analog of the problem presented here as a possible extension of the model they study.

\subsection{Analysis}

To focus on the communication issue, I start by assuming that the agent chooses the investment allocation which maximizes the weighted average of performance measures communicated by the center. Later, I will show how the main results change when the center must provide some incentive to motivate the agent (under moral hazard). For now, the agent maximizes the weighted sum of performance measures (including the communicable part of the strategy) $\beta^{0} v^{0}+\sum_{j=1}^{J} \beta^{j} E m^{j}$, with $\left(\beta^{0}, \beta^{1}, \ldots, \beta^{J}\right)$ being the vector of linear weights on the communicable strategy and on the performance measures,

$$
\max _{q(\theta)} E\left(\beta^{0} v^{0}(\theta, q(\theta))+\sum_{j=1}^{J} \beta^{j} m^{j}(\theta, q(\theta))\right)
$$

The first order conditions of the agent's problem are, ${ }^{9}$

$$
\beta^{0} v_{q}^{0}(\theta, q(\theta))+\sum_{j=1}^{J} \beta^{j} m_{q}^{j}(\theta, q(\theta))=0 \quad \text { if } q(\theta)>0,
$$

The agent has the important responsibility of selecting the projects. The agent invests only in the projects for which the marginal return on the performance measures, or performance gains, is positive.

$$
q(\theta)>0 \Leftrightarrow \beta^{0} v_{q}^{0}(\theta, 0)+\sum_{j=1}^{J} \beta^{j} m_{q}^{j}(\theta, 0)>0 .
$$

Without loss of generality, I will assume that all projects are selected, $\beta^{0} v_{q}^{0}(\theta, 0)+$ $\sum_{j=1}^{J} \beta^{j} m_{q}^{j}(\theta, 0)>0, \forall \theta \in \Theta$. The center computes the agent's response function $q\left(\theta,\left(\left(\beta^{0}, \beta^{1}, \ldots, \beta^{J}\right)\right)\right.$ given the performance weights using the agent's first order conditions (2). The agent's response function is homogeneous of degree zero in $\left(\beta^{0}, \beta^{1}, . ., \beta^{J}\right)$.

\footnotetext{
${ }^{9}$ The second order conditions require that $\beta^{0} v_{q q}^{0}+\sum_{j=1}^{J} \beta^{j} m_{q q}^{j} \leq 0$. This condition will hold at the optimum because $\beta \geq 0$.
} 
In other words, only relative weights matter, so that if $\left(\beta^{0}, \beta^{1}, . ., \beta^{J}\right)$ implements a given investment then any scalar multiple of $\left(\beta^{0}, \beta^{1}, \ldots, \beta^{J}\right)$ also implements this investment. Therefore, one can focus on the relative performance weights only and ignore the absolute level of weights. For simplicity, I will assume without loss of generality that $\beta^{0}=1$ and I will note $\beta=\left(\beta^{1}, \ldots, \beta^{J}\right)$. The agent response function is,

$$
q_{\beta}=\Leftrightarrow\left(v_{q q}^{0}+\beta^{\prime} m_{q q}\right)^{-1} m_{q} .
$$

Investment allocation $\tilde{q}(\theta)$ is feasible if there exists a set of weights $\beta$ such that $\tilde{q}(\theta)=$ $q(\theta, \beta)$. The center chooses the feasible allocation which maximizes the average value over all projects.

$$
\max _{\beta} E v(\theta, q(\theta, \beta))
$$

The center's first order conditions are given by,

$$
E v_{q}(\theta, q(\theta, \beta)) q_{\beta}(\theta, \beta)=0 .{ }^{10}
$$

The center's $J$ first order conditions in addition to the agent's first order conditions give the solution to the optimal weighting scheme $\beta$ and to the optimal investment decisions $q(\theta, \beta)$. Plugging the value of $q_{\beta}$ in equation 5 , the center's first order conditions can be written as,

$$
E v_{q}\left(v_{q q}^{0}+\beta^{\prime} m_{q q}\right)^{-1} m_{q}=0
$$

$v_{q}$ will be interpreted as the value-added to the organization while $m_{q}$ will be interpreted as the performance gains. To a first order approximation $\left(v_{q q}^{0}\right.$ and $m_{q q}$ are constant), this result states that the value-added is orthogonal to the performance gains. This is intuitive because the measures are used to minimize the investment mistakes which are measured at the margin by the value-added to the organization. After replacing from the agent's first order condition the communicable part of the organization's strategy in the above equation,

$$
\beta=\left(E m_{q}\left(v_{q q}^{0}+\beta^{\prime} m_{q q}\right)^{-1} m_{q}^{\prime}\right)^{-1} E m_{q}\left(v_{q q}^{0}+\beta^{\prime} m_{q q}\right)^{-1} v_{q}^{1} .
$$

\footnotetext{
${ }^{10}$ The second order conditions to the center's problem hold under some general assumption on the third derivative of the measure. This is shown in the Appendix.
} 
By abuse of notation, we will interpret $v_{q}^{1}$ as value-added although it represents only the part of value-added that the organization is trying to communicate. This leads to the following proposition.

Proposition 1 (1) There exists a diagonal weighting matrix $P=v_{q q}^{0}(\theta, q(\theta))+\beta^{\prime} m_{q q}(\theta, q(\theta))$ s.t. $\beta$ is the regression coefficient of the value-added to the organization on the performance gains with weighting matrix $P,{ }^{11}$

$$
\beta=\left(E m_{q} P m_{q}^{\prime}\right)^{-1} E m_{q} P v_{q}^{1}
$$

(D) To a first order approximation $\left(v_{s s}^{0}(\theta, q(\theta))=v_{s s}^{0}\right.$ and $\left.m_{s s}(\theta, q(\theta))=m_{s s}, \forall \theta \in \Theta\right)$, the optimal linear weights equal the regression coefficient of the value-added to the organization on the performance gains.

$$
\beta=\left(E m_{q} m_{q}^{\prime}\right)^{-1} E m_{q} v_{q}^{1}
$$

This result deserves some attention. Measures of organization's value and performance outcome do not matter; only value-added and performance gains matter. This result is intuitive since the organization really cares about value-added and the agent responds only to performance gains. The center puts more weights on the performance measures which better predict value-added on the part of the strategy which cannot be communicated. This is also a fairly intuitive result. The agent selects the projects with high return on the linear combination of performance measures. These projects are also the most valuable for the organization when at the margin, the communicated objective approximates the true strategy well. More surprising, however, is that the dimension of the strategy which can be communicated plays no role (at least in a first order approximation sense) in the formula for the optimal performance weights. In this sense, the communication problem is separable: the center communicates to the agent only what she does not know without distorting what she knows.

Interestingly, the optimal weights have an econometric interpretation, despite the fact that there is no uncertainty in this model. The organization can be seen as bridging the

\footnotetext{
${ }^{11}$ The matrix $P$ can be interpreted as a correction matrix for heteroskedasticity.
} 
informational gap by solving an optimal decision problem under uncertainty. Linear least squares turn out to be the optimal solution to this problem. The optimal weights are chosen because they perform well on average. Notice that the regression interpretation suggests that the correlation between performance gains and value-added is not always a good proxy for the performance weights. For a given measure, the correlation is a good proxy when (a) there exists a performance measure which is linear in the investment decision; and (b) the performance measures are independent.

Imperfect performance measures addresses the communication problem but distort the investment decisions. Let $q^{*}(\theta)$ be the efficient level of investment for project $\theta$. At the efficient level of investment, there are no more opportunities for adding values,

$$
v_{q}\left(\theta, q^{*}(\theta)\right)=0
$$

The center achieves the first-best outcome if and only if there exists a $\beta \in \Re^{J}$ such that $v_{q}^{0}\left(\theta, q^{*}(\theta)\right)+\beta^{\prime} m_{q}\left(\theta, q^{*}(\theta)\right), \quad \forall \theta \in \Theta$. In general, the first-best conditions do not hold because these conditions would require the center to control a very large number of investment decisions but she only controls $J$ instruments. ${ }^{12}$ As a consequence, the valueadded is positive for some projects $\left(v_{q}>0\right)$ and negative for other projects $\left(v_{q}<0\right)$. Under the optimal performance weights, the center makes some trade-offs between projects, overinvests in some and under-invests in others.

To conclude this section, notice that the above formula has some empirical implications that could be tested. Although the informational gap assumption states that it is too costly for the organization to communicate its strategy to the agent on a systematic basis it does not rule out the possibility to collect data on the value-added to the organization and on the performance gains for a restricted set of projects. Actually, these data are sometimes produced for internal business evaluation or for more global evaluation at the organization-level. In an empirical application, one could estimate the optimal balance of

\footnotetext{
${ }^{12}$ The comparison of interest is between $\operatorname{dim}(\Theta)$ and $J$. If there is a finite number of projects $\operatorname{dim}(\Theta)=$ $I$, the first-best requires $I$ first order conditions and the center controls $J$ dimensions. As long as $I>>J$, the conclusions presented in the text follow.
} 
performance measures for a restricted representative sample of projects and compare these theoretical weights with the observed weights. ${ }^{13}$ Using the information on the estimated performance weights one can answer the questions: (1) Do the performance measures used in practice predict value-added by testing, if the coefficients on the measures are significantly different than zero (and have the predicted sign)? (2) Do the performance measures provide the right incentives by testing whether the ratio of two performance weights equals the predicted ratio?

\subsection{Discussion: The Strategic Role of Performance Measures}

Although this model can be applied to many organizational contexts, this section focuses on an application to strategic management because the communication feature of performance measures has recently received much attention in this context. Consultants offer a wide range of performance metrics to measure how much 'economic value' a company creates. These metrics are usually not based on accounting measures which are easy to manipulate, but rather on cash flow. For example, economic value-added (EVA), popularized by Bennett Stewart (1991) deducts from a firm's earnings a charge for the different amount of capital (such as bonds, equity, loans) it employs. A very stylized version of EVA looks like,

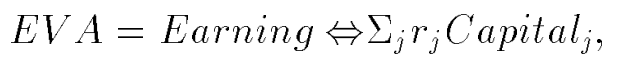

where $r_{j}$ is the cost of capital $j$. These measures were originally developed as an alternative thermometer of corporate performance to shareholder value for financial investors but they are now transformed into more forward looking corporate-governance tools to persuade managers to act in line with their owners. Compared with those more general performance metrics, shareholder value has at least two important drawbacks: (a) it does not focuses the organization on an explicit and transparent goal and more importantly, (b) it cannot

\footnotetext{
${ }^{13}$ Notice that the econometrician observes only those projects which have been selected. However, a generalization of formula (8) to the case where the agent selects the projects would also take into account only these projects. Therefore, the econometrician estimates the right formula by using the sample of selected projects.
} 
be driven vertically into each functional organization, whereas it can be broken down into its component parts in order to evaluate how each part contributes to the functioning of the whole.

The main advantage of EVA is that it focuses managers on adding value to their companies by increasing earnings, by reducing the amount of capital employed, or by decreasing the cost of capital. Some firms believe that value-based management is a good performance driver and have tied executives' share options and/or bonuses to improvements in these performance measures. EVA, however, is just one out of many performance metrics combining the pure measurement of corporate performance aspect with a strategic management dimension (The Economist, 1997). The common focus on the design of all these new measures is how well they will drive senior managers in each major organizational unit as well as frontline managers and employees to assess the impact of their activities on the entire company's valuation.

The balance scorecard goes one step further and combines financial scores with measures of less tangible assets such as customer satisfaction and loyalty (sometimes called customer value-added), and a firm's ability to nurture the skills of its employees (Kaplan and Norton, 1993). More largely defined performance measures give a remedy to the limitations of measuring only short-term financial results. Under this broader view, performance measures make the change from financial to strategy reporting. A measurement system is viewed as a management system to translate coherent business strategies and to motivate breakthrough competitive performance.

According to this recent trend toward a more strategic use of measurement system, organizations not only introduce new measures to communicate new strategies but also question whether old measures are relevant to new initiatives. This prediction that organizations should respond to change in strategy is entirely consistent with the communication role of performance measures. The simple regression formula implies that organizations should change their measurement systems in response to a change in their strategies (a change in $v$ in the model.) Interestingly, this prediction contrasts with the prediction 
that would follow under the alternative interpretation of performance measures as pure motivational devices (e.g. piece rate or sharing rules) to induce the agent to exert effort. Under an incentive interpretation, performance measures are control instruments rather than strategic tools and do not depend so much on the organization's strategy.

\section{How Do Performance Measures Perform?}

Although the previous section showed how organizations select and balance performance measures, it did not establish when performance measures are good communication devices. The evaluation of how performance measure perform is a difficult one. To address this question, I compare the performance of performance measures with two informative benchmarks. First, to evaluate the absolute advantage of performance measures, I compare their performance with the first-best performance where there is no informational gap in the organization. Second, to evaluate the relative advantage of performance measures, I compare their performance with an alternative second-best organization; a commandand-control organization, where agents have no discretion over the investment decisions.

I conclude this section by showing that more local information might not improve the performance of measurement systems.

\subsection{The Absolute Advantage of Performance Measures}

To study the distortion due to imperfect communication, I compare the value generated by a performance measure-driven organization with the hypothetical one generated under perfect communication. To simplify this comparison analysis, it is convenient to make a second order approximation of value functions around the range of $q(\theta)$ as it varies within the optimal level of investment $q^{*}(\theta)$,

$$
v^{i}(\theta, q(\theta))=v^{i}\left(\theta, q^{*}(\theta)\right)+\left(q(\theta) \Leftrightarrow q^{*}(\theta)\right) v_{q}^{i}\left(\theta, q^{*}(\theta)\right)+\frac{1}{2}\left(q(\theta) \Leftrightarrow q^{*}(\theta)\right)^{2} v_{q q}^{i},
$$

for $i=1,2$, where $v_{q q}$ is a constant. Differentiating the second order approximation (11) with respect to $q(\theta)$, adding the two approximations and replacing the agent's first order 
conditions (2) gives,

$$
q(\theta)=q^{*}(\theta)+\frac{1}{v_{q q}} R\left(v_{q}^{1} \mid m_{q}\right),
$$

where $R\left(v_{q}^{1} \mid m_{q}\right)=v_{q}^{1}(\theta, q(\theta)) \Leftrightarrow \beta^{\prime} m_{q}(\theta, q(\theta))$ stands for the residual of the regression of value-added on performance gains. Equation (12) shows how imperfect performance measures distort the investment allocation. The sign of the distortion depends on the sign of the residual of the regression of value-added on performance gains. The agent under-invests (over-invests) in the projects whose value-added has been under-estimated (over-estimated). The level of distortion is proportional to the error in the estimation of value-added. The coefficient of proportionality is equal to a measure of curvature in the value functions. Curvature in the value functions tends to reduce the investment distortions. More curvature around the optimal benefit implies that, for a given change in the investment, value-added varies more. Therefore, the investment decisions are less sensitive to errors in the estimated value-added.

One can compute the total loss in revenue due to the use of imperfect performance measures. Let $v(m)$ and $v^{*}$ be the optimal value generated under a performance measuredriven organization and under the efficient investment. Replacing $q(\theta) \Leftrightarrow q^{*}(\theta)$ from equation (12) in equations (11) gives,

$$
v^{*} \Leftrightarrow v(m)=\frac{\Leftrightarrow 1}{2 v_{q q}} E R^{2}\left(v_{q}^{1} \mid m_{q}\right)
$$

Better proxies for the value function reduce the cost of using imperfect performance measures. The performance of performance measures depends proportionally on the share of the variations in value-added explained by the performance measures, and inversely proportionally on the curvature of the value function.

This model offers a simple method to evaluate the value of using a set of performance measures. Assume an organization can use two sets of performance measures, $m$ and $\tilde{m}$. The change in value due to a switch from performance measures $m$ to $\tilde{m}$ is,

$$
v(m) \Leftrightarrow v(\tilde{m})=\frac{\Leftrightarrow 1}{v_{q q}} E\left(R^{2}\left(v_{q}^{1} \mid \tilde{m}_{q}\right) \Leftrightarrow R^{2}\left(v_{q}^{1} \mid m_{q}\right)\right)
$$


In other words, an organization is willing to pay (or must be compensated) to switch from the set of performance measures $m$ to the set $\tilde{m}$ an amount proportional to the change in the variation in value-added explained by these two sets.

Notice that the organization cannot decreases value by combining more performance measures together. (The center always have the option not to weight the measures which do not have any explanatory power.) However, focusing on the benefit side only ignores that there are also costs to using performance measurement systems. In practice, at least two factors limit the number of performance measures. The first factor is comprehension. It has been repeatedly shown that people have difficulty understanding complex measurement systems (Lawler (1990)). Second, there are important costs to collect performance outcomes and to maintain and perfect measurement systems (Burns and McKinnan, 1992).

A final point can be made on how the benefit of using performance measures changes with the size of an organization. Ceteris paribus, performance measures are more valuable in larger organizations. To see this, assume a continuum of organizations identified by

their size $\lambda$ such that $\tilde{v}_{\lambda}(\theta, q)=\lambda v(\theta, q)$. The efficiency loss of using imperfect performance measures is homogeneous of degree one with the organization's scale in the sense that

$$
v_{\lambda}^{*} \Leftrightarrow v_{\lambda}(m)=\lambda K
$$

where $K$ is a constant equal to $\left.v_{1}^{*} \Leftrightarrow v_{1}(m)\right)$. As a consequence, larger organizations should spend more resources collecting performance measures because the opportunity gain of additional performance measures is larger in these organizations. This may explain why more mature organizations which tend to be larger are more likely to develop and invest in measurement systems and control systems more generally defined.

\subsection{The Comparative Advantage of Performance Measures}

To guide and improve local decision making, some organizations use measurement systems while others use other devices. The issue of when one should observe performance 
measures rather than an alternative device is a 'second-best' question. Although the comparison with the 'first-best' organization told us what may go wrong when performance measures are used, it misses the point of establishing a criterion for when performance measures should be observed. An insightful comparison should be a comparison with a competing 'second best' organizational design. A naturally competing organizational design is one which uses an alternative low-cost communication device. Following Weitzman (1971), the value generated by a central command-and-control top-down organization which fixes the same investment rule for all the projects will be compared with the value generated by a performance measures driven organization. ${ }^{14}$

In a top-down organization, the agent's private information of the projects is not used. The agent is asked to go through fixed routines for each project. There is a priori no reason why one organizational design should generate more value than the other. The command-and-control organization chooses investment $\bar{q}$ such that,

$$
E v_{q}(\theta, \bar{q})=0
$$

Following Weitzman (1974), let $\Delta$ be the difference in value between the performance measure-driven organization and the fixed-investment organization,

$$
\Delta=E(v(\theta, q(\theta)) \Leftrightarrow v(\theta, \bar{q}))
$$

As previously, a second order approximation is made of the value functions but now within the range of $q(\theta)$ as it varies around $\bar{q}$,

$$
v^{i}(\theta, q(\theta))=v^{i}(\theta, \bar{q})+(q(\theta) \Leftrightarrow \bar{q}) v_{q}^{i}(\theta, \bar{q})+\frac{1}{2}(q(\theta) \Leftrightarrow \bar{q})^{2} v_{q q}^{i}
$$

for $i=0,1$. Differentiating the second order approximation and rearranging,

$$
q(\theta) \Leftrightarrow \bar{q}=\frac{R\left(v_{q}^{1} \mid m_{q}\right) \Leftrightarrow \epsilon_{v_{q}(\theta, \bar{q})}}{v_{q q}}
$$

\footnotetext{
${ }^{14}$ Weitzman assumed a single performance measure: the quantity produced. (Using this paper's notations, $m(\theta, q)=q$.) This particular functional form turns out to be crucial for his results.
} 
where $\epsilon_{v_{q}(\theta, \bar{q})}=v_{q}(\theta, \bar{q}) \Leftrightarrow E v_{q}(\theta, \bar{q})$. Plugging (17) into (16) gives the expression for the coefficient of comparative advantage,

$$
\Delta=\frac{\Leftrightarrow 1}{2 v_{q q}}\left(\sigma_{v_{q}(\theta, \bar{q})}^{2} \Leftrightarrow E R^{2}\left(v_{q}^{1} \mid m_{q}\right)\right)
$$

where $\sigma_{x}^{2}$ stands for the variance of $x$. Notice that the variance in the value function is measured at $\bar{q}$ while the expected residual square error is measured at $q(\theta)$. The performance-measure organizational regime dominates the command-and-control organizational regime when ceteris paribus: (1) The agent observes more local information on how value-added varies across projects $\left(\sigma_{v_{q}(\theta, \bar{q})}^{2}\right.$ is high). The variance in value-added is a measure of the agent's knowledge which is not used under the fixed-investment regime. (2) The performance gains do not create too many dysfunctional responses. When this occurs, the residual square error of the regression of value-added on performance gains is small.

\subsection{The Return on Local Information}

Local information is valuable when it helps the agent to make decisions congruent with the organization's strategy. This observation suggests that more local information may not always be valuable. A simple example illustrates how more local information may actually lower value. Assume two projects and one performance measure. The value function is the same for both projects $v(\theta, q)=3 \Leftrightarrow(1 \Leftrightarrow q)^{2}, \theta=1,2$ but the performance measure is not: $m(1, q)=\Leftrightarrow q^{2}$ and $m(2, q)=\Leftrightarrow(2 \Leftrightarrow q)^{2}$. If the agent can identify each project, she invests no resource in the first project $(q(1)=0)$ and two units in the second $(q(2)=2)$. However, if the agent cannot discriminate between the projects she maximizes $m(1, q)+m(2, q)$ and chooses the efficient investment for both projects $(q(1)=q(2)=1)$. In this example, local information reduces the organization's value because the marginal information does not represent changes in value-added.

This observation sheds some light on a fundamental flaw of rewarding high and punishing low performances resulting in agent behavioral responses which are sometimes called 
gaming (Lawler 1990). Once an measurement system is put in place, the incentives created do not depend any more on the motivation which led to them. Self-interest motivates the agent to collect local information in order to understand the performance measures on which his rewards and punishments depend. It does not take the agent long to discover where the rewarded objective is different from the stated one. The agent uses his superior local information about how his actions influence his performance award. Since the performance measures do not always give the agent accurate incentives, the agent engages in actions that the organization, if it had the agent's information, would consider non-optimal. ${ }^{15}$

Gaming has been demonstrated in several organizational environments. In the context of managerial compensation, Healy (1985) showed that managers manipulate reporting variables to maximize their inter-temporal awards. Oyer (1996) presents similar results in the context of sales force compensation. Courty and Marschke (1997) demonstrate gaming in the context of a government bureaucracy, by conducting a formal efficiency analysis.

\section{$4 \quad$ Extensions}

\subsection{Absolute and Relative Weights}

The balancing formula for the relative performance weights derived in Subsection 2.2 overlooks the fact that measurement systems are sometimes coupled with incentive systems. One may wonder whether the interpretation of the performance weights as a regression formula is still valid once the incentive dimension is taken into account. To answer this question, I extend the model to allow for the role of absolute incentive weights in providing rewards.

The standard model of incentives is the principal-agent model of moral hazard first

\footnotetext{
${ }^{15}$ This interpretation of gaming is slightly different than the one found in Baker (1992). In Baker's model, gaming occurs essentially because performance gains do not represent well value-added. The type of gaming identified here is much stronger: the agent may collect counter-productive information that result in negative value-added.
} 
formally developed by Holmstrom (1979). In this model, moral hazard problems prevent the principal from observing the effort exerted by a risk-adverse agent. The principal only observes some noisy proxies for the agent's effort. As a consequence, the principal must make a trade-off between providing incentives to exert effort and having the agent bare unnecessary risks. Following the standard approach, I assume that the performance measures are noisy in the sense that the observed outcome $\tilde{m}$ is,

$$
\tilde{m}=\operatorname{Em}(\theta, q(\theta))+\epsilon
$$

with epsilon a zero-mean normal vector with variance-covariance matrix $\Sigma$. As usual, this matrix will be interpreted as measurement noise. The agent is risk averse with utility function $u(c)=\Leftrightarrow e^{-r w} \cdot q(\theta)$ is interpreted as the agent's level of effort for project $\theta$ and $v^{0}(\theta, q(\theta)$ as her cost of effort. There is moral hazard because the principal cannot contract on $v^{0}$.

Following the standard approach (Gibbons, 1996), the organization maximizes the sum of her certainty equivalent and the one of the agent,

$$
\max _{q(\theta, \beta), \beta} \operatorname{Ev}(\theta, q(\theta, \beta)) \Leftrightarrow \frac{r}{2} \beta^{\prime} \Sigma \beta .
$$

One can easily solve for the optimal weights,

$$
\beta=\left(E m_{q}\left(v_{q q}^{0}+\beta^{\prime} m_{q q}\right)^{-1} m_{q}^{\prime}+r \Sigma\right)^{-1} E m_{q}\left(v_{q q}^{0}+\beta^{\prime} m_{q q}\right)^{-1} v_{q}^{1} .
$$

As expected, this formula is equivalent to formula (7) if the agent is risk neutral $(r=0)$ or if the measures are not noisy $(\Sigma=0)$. More noisy measures have the well-studied effect of decreasing the incentive weights. Similarly, this formula is equivalent to the one found in the principal-agent literature when the performance measures do not explicitly communicate a strategy, $m(\theta, q)=m(q)$. More generally, however, the projection formula is a good approximation for the weights as long as the measurement noise is small, relative to the information communicated by the measures ( $\Sigma$ small relative to $E m_{q} m_{q}^{\prime}$.)

Actually, the importance of the communication role of performance measures becomes more striking once the principal-agent model is modified to be consistent with the environments in which measurement systems are commonly observed. The case considered 
in the standard principal-agent literature is an extreme case where the agent supplies (through his effort) all the resources invested in the projects. This overlooks the fact that in most organizational contexts, the agent's effort represents only a very small part of total investment. The organization provides most the investment in terms of human resources (support staff, delegation), capital resources (information systems, durable investments) and financial resources. Taking this observation into account asks for a revision of the standard formulation. Assume the agent's cost of effort represents only a fraction $k$ of total investment. (For example, $k=0.08$ if a manager is paid $\$ 40,000$ per year and controls a budget of $\$ 500,000 .{ }^{16}$ ) Given performance award $\beta^{\prime} m$, the manager chooses investments to maximize $k v^{0}+\beta^{\prime} m$, while the organization maximizes the same objective as before. This leads to the following proposition.

Proposition 2 As the fraction of the agent's investment in the projects become arbitrarily small, the absolute incentive weights also become arbitrarily small and the relative performance weights converge to the pure communication performance weights.

Proof: See Appendix.

This proposition is fairly intuitive. It states that as the agent's personal investment becomes small relative to total investment, the performance measures are really used as a communication device rather than as an incentive device. In contrast with Holmstrom and Milgrom (1991), this proposition explains why low powered incentive systems are often observed in practice by showing that although low powered incentives have little use in term of increasing the agent's effort contribution, they can be quite useful in term of channeling existing resources. Low powered incentive perform the important economic role of improving local decision making by communicating a clear goal.

This proposition also suggests that incentives are more likely to be high powered when the agent's investment accounts for a large fraction of total investment. An implication,

\footnotetext{
${ }^{16}$ Actually, this overestimates the importance of the agent's unobserved effort (under moral hazard) since a fixed salary usually accounts for most of total compensation.
} 
for example, is that a piece rate perform well when the agent can be charged for the raw inputs, and when she owns the means of production. Under these assumptions, the agent's investment accounts for a large fraction of total investment. ${ }^{17}$ This prediction, however, is similar to the one found in the standard principal-agent literature (Holmstrom and Milgrom, 1991).

To summarize, the weighting formulas derived under a pure communication model $(\Sigma=0)$ and the one derived under the principal-agent model $(m(\theta, q(\theta))=m(q))$ correspond to two different interpretations of measurement systems: communicating a strategy and inducing risk averse agents to exert effort. In the standard principal-agent paradigm, the relative performance weights depend to a large extend on the variance-covariance matrix of the measurement errors, or on the statistical concept of sufficient statistics under more general assumptions on the distribution of these errors. Since little can be known in practice about this variance-covariance matrix, the principal-agent model has little to say about the relative performance weights. In contrast, the model presented here provides a simple and intuitive formula for the choice and balance of performance measures by distinguishing and isolating the communication and incentives roles of these measures.

\subsection{Divisional Performance Evaluation}

Divisional performance evaluation distinguishes profit, cost, and benefit centers (Brickley, Simth, and Zimmerman, 1996). These evaluation systems correspond to different strategies communicated by the organization's head quarter to its divisions. I first ask whether the organization can separately communicate the cost and benefit function to the agent through two independent sets of cost and benefit performance measures. Then I study when the organization chooses to communicate one of these objective rather than the other.

To study the factors determining the choice of divisional performance evaluation, the

\footnotetext{
${ }^{17}$ Note also that an additional requirement for a piece rate to perform well is that quality be easily assessed. Quality can be interpreted in this model as being easily assessed when performance gains represent well value-added.
} 
value function is decomposed into a benefit and a cost function $v^{1}=b \Leftrightarrow c$. Assume the center independently communicates these two functions through two sets of performance measures: a set of proxies for the cost functions $m^{c, j}(\theta, q) ; j=1 \ldots J^{c}$ and a set of proxies for the benefit functions $m^{b, j}(\theta, q) ; j=1 \ldots J^{b}$. Assume the cost proxies are increasing and convex and the benefit proxies are increasing and concave. Let $\beta^{c}$ and $\beta^{b}$ be the weights on the cost and benefit measures. When $m_{q q}^{b}$ and $m_{q q}^{c}$ are constant, the optimal performance weights correspond to the regression weights of benefit and cost value-added on benefit and cost performance gains.

$$
\left(\begin{array}{c}
\beta^{b} \\
\beta^{c}
\end{array}\right)=E^{-1}\left(\begin{array}{c}
m_{q}^{b} \\
m_{q}^{c}
\end{array}\right)\left(\begin{array}{c}
m_{q}^{b} \\
m_{q}^{c}
\end{array}\right)^{\prime} E\left(\begin{array}{c}
m_{q}^{b} \\
m_{q}^{c}
\end{array}\right) v_{q}^{1}
$$

Assume the benefit measures are independent of the cost measures at the margin $\left(c_{q}(\theta, q), m_{q}^{c}(\theta, q)\right) \perp\left(b_{q}(\theta, q), m_{q}^{b}(\theta, q)\right)$. This assumption is supported by the observation that the random factors which influence the value of the projects are independent from the random factors which influence the costs of the inputs. Quite surprisingly, this assumption does not guaranty separability in the communication problem. That is, the weights on the benefit (cost) measures may not be the regression weights of benefit (cost) value-added on benefit (cost) performance gains. This is because at the optimum investment level, the benefit performance gains and the cost performance gains are not independent. They must satisfy the agent first order conditions,

$$
v_{q}^{0}(\theta, q(\theta))+\beta^{b^{\prime}} m_{q}^{b}(\theta, q(\theta))+\beta^{c^{\prime}} m_{q}^{c}(\theta, q(\theta))=0 .
$$

Because the cost and benefit performance gains are in general statistically dependent (through the choice of the optimal investment level $q(\theta)$ ), it is more efficient to aggregate the information jointly over both cost and benefit measures rather than separately. In general, one can show that the regression formula is separable when the cost and benefit functions are linear in the cost and benefit measures $\left(b_{q}=\beta^{b^{\prime}} m_{q}^{b}+\eta^{b}\right.$ with $\eta^{b}$ an independent noise vector and similarly for the cost measures.) If this linearity assumption holds, the cost of using imperfect performance measures is separable in two components: 
the cost of misrepresenting the benefit functions, and the cost of misrepresenting the cost functions,

$$
E R^{2}\left(v_{q}^{1} \mid m_{q}^{b}, m_{q}^{c}\right)=E R^{2}\left(b_{q} \mid m_{q}^{b}\right)+E R^{2}\left(c_{q} \mid m_{q}^{c}\right)
$$

Under the separability assumption, we can identify important differences between cost, benefit and profit centers. A cost center minimizes costs of producing a fixed quantity given a set of shadow input prices communicated by the center. The cost center takes into account only cost measures meaning that it has much local information on the optimal input mix given the shadow prices of input, and either knows the true benefit function or observes no relevant information about it. Similarly, a profit center ignores both the cost and benefit functions and observes local knowledge influencing these two. More generally, the organization communicates the dimension of its true strategy that the agent ignores and about which the agent observes relevant local information.

\section{$5 \quad$ Summary and Conclusions}

This paper interprets measurement systems as imperfect mechanisms which improve local decision-making within organizations by communicating clear objectives. Within this framework, I explain how organizations choose performance measures and how they balance these measures to communicate their strategies. The optimal balance of performance measures has a regression interpretation where the organization combines the measures in a way that performance gains come closest to mimicking value-added to the organization. The organization places more weight on the performance measures which better predict value-added. In response, the agent selects and invests more in the projects with a higher return on the combination of performance measures which are also, one average, more profitable for the organization.

This view of measurement systems as communication devices contrasts with their traditional view as incentive devices. Under this communication interpretation, these systems perform an economic role even in the absence of rewards or punishments for high or low performances: these systems induce agents to better allocate the organization's 
resources. This is consistent with the observation that many measurement systems, or control systems more generally viewed, do not offer any financial rewards. This framework also captures the view that effective measurement is becoming an integral part of the management process where companies translate their strategies into a coherent set of performance measures. In some contexts, however, performance measurement systems play the dual role of communicating objectives and of inducing effort. One of the main contribution of this paper is to distinguish these two roles and to show that the communication interpretation leads to different predictions for how these systems should be constructed and used.

From an empirical standpoint, this paper suggests several empirical ways to evaluate the practical importance of the communication role of measurement systems. To close this discussion, I briefly summarize how these predictions contrast with those derived under the standard principal-agent model of performance incentives found in the organization literature. First, this paper implies that the gains from performance measurement systems are greater in larger organizations while the principal-agent model suggests these gains are independent of the organization's size. Second, the interpretation of performance measures as a communication device implies that organizations should not only introduce new measures to motivate new goals but also need to question whether their old measures are relevant to new initiatives. Therefore, one should find within an organization a close relationship between changes in strategy and changes in measurement systems. Finally, the organization literature has focused on tests of absolute incentive weights and has overlooked the communication role in determining relative incentive weights. This work shows that under a pure communication assumption, the relative performance weights depend on the relationship between performance gains and value-added but not on the measurement noise in value-added as is predicted under a pure incentive assumption. This difference in the formulas could be tested against real data. 


\section{Appendix}

\section{Second Order Conditions}

The second order conditions to problem (4) are given by,

$$
E\left(v_{q q} q_{\beta} q_{\beta}^{\prime}+v_{q} q_{\beta \beta^{\prime}}\right)
$$

Let's compute $q_{\beta \beta^{\prime}}$. Differentiating the agent's first order conditions with respect to $\beta$ and rearranging terms,

$$
q_{\beta \beta^{\prime}}=\left(\beta^{\prime} m_{q q}\right)^{-2}\left(2 m_{q q} m_{q}^{\prime}-\beta^{\prime} m_{q q q}\left(\beta^{\prime} m_{q q}\right)^{-1} m_{q} m_{q}^{\prime}\right) \text {. }
$$

Let $M$ be the matrix of second order conditions. Plugging this expression in (21),

$$
M=E\left(\beta^{\prime} m_{q q}\right)^{-2}\left(\left(v_{q q}-v_{q} \frac{\beta^{\prime} m_{q q q}}{\beta^{\prime} m_{q q}}\right) m_{q} m_{q}^{\prime}+2 v_{q} m_{q q} m_{q}^{\prime}\right) .
$$

The second order conditions require that the matrix $M$ be negative semi-definite.

\section{Proof of Proposition 2}

Replacing the agent first order condition in the principal first order conditions gives,

$$
\beta=k\left(E m_{q}\left(k v_{q q}^{0}+\beta^{\prime} m_{q q}\right)^{-1} m_{q}^{\prime}+k r \Sigma\right)^{-1} E v_{q}^{1}\left(k v_{q q}^{0}+\beta^{\prime} m_{q q}\right)^{-1} m_{q}
$$

Taking a first order approximation,

$$
\frac{\beta}{k}=\left(E m_{q} m_{q}^{\prime} \Leftrightarrow k r\left(k v_{q q}^{0}+\beta^{\prime} m_{q q}\right) \Sigma\right)^{-1} E v_{q}^{1} m_{q} .
$$

$\beta$ is bounded as $k$ goes to zero; otherwise the cost of insurance would be unbounded which cannot be an optimum. Therefore, $\beta$ converges to zero as $k$ goes to zero. Finally, the relative performance weights $\frac{\beta}{k}$ converge to the linear least square approximation,

$$
\frac{\beta}{k}=\left(E m_{q} m_{q}^{\prime}\right)^{-1} E v_{q}^{1} m_{q}
$$




\section{References}

1. Baker, George P., Jensen, Michael C., and Murphy, Kevin J. "Compensation and Incentives: Practice vs. Theory." The Journal of Finance 43 (1988): 593-615.

2. Baker, George P. "Incentive Measures and Performance Measurement." Journal of Political Economy, 100 (1992): 598-614.

3. Brickley, James, Cliffort Simth and Jerold Zimmerman. Managerial Economics and Organizational Architecture. Irwin, 1996.

4. Burns, William and Sharon McKinnon. "Performance Evaluation and Managers' Descriptions of Tasks and Activities." In Performance Measurement, Evaluation, and Incentive, edited by W. Burns. Harvard Business School Press, 1992.

5. Courty, Pascal and Gerald Marschke. "Measuring Government Performance: Lessons from a Federal Job-Training Program." American Economic Review, 87 (2): 383388.

6. Eccles, Robert. "The Performance Measurement Manifesto." Harvard Business Review, January-February 1991, p. 131-7.

7. Gibbons, Robert. "Incentives and Careers in Organizations." Johnson Graduate School of Managment. Unpublished Manuscript, 1996.

8. Hayek, Frederich A. von. "The Use of Knowledge in Society." American Economic Review, 35 (1945): 519-530.

9. Healy, Paul M. "The Effect of Bonus Schemes on Accounting Decisions." Journal of Accounting and Economics , 7 (1985): 85- 107.

10. Holmstrom, Bengt. "Moral Hazard and Observability." The Bell Journal of Economics, 10 (1979): 74-91.

11. Holmstrom, Bengt, and Milgrom, Paul. "Multitask Principal- Agent Analyses: Incentive Contracts, Asset Ownership, and Job Design." The Journal of Law, Economics, and Organization , 7 (1991): 24-52.

12. Jensen, Michael and William Merckling. Specific and General Knowledge, and Organizational Structure. In Contract Economics, edited by Lars Werin and Hans Wijkander. Basic Blackwell, Oxford, 1992.

13. Kaplan, Robert and David Norton. 'Putting the Balanced Scorecard to Work." Harvard Business Review, (September-October 1993):134-147.

14. Kaplan, Robert and David Norton. The Balanced Scorecard. Harvard Business School Press. Boston, Massachusetts, 1996. 
15. Locke, Edwin and Gary Latham. A Theory of Goal Setting and Task Performance. Prentice-Hall, Englewook Cliffs, NJ: 1990.

16. Lawler, Edward E. III. Pay and Organizational Strategies and Pay Systems. JosseyBass, San Francisco: 1990.

17. Oyer, Paul. "The Effect of Sales Incentives on Business Seasonality." Mimeo. Princeton University. 1996.

18. Rajagopalan, Nandini. "Strategic Orientations, Incentive Plan Adoptions, and Firm Performance: Evidence from Electric Utility Firms." Strategic Management Journal. Vol. 18, (1996):761-785.

19. Stewart, Bennett. Quest for Value. Harper Collins, New York: 1991.

20. Weitzman, Martin L. "Prices vs Quantities." Review of Economic Studies, (1974): 477-91. 